\title{
INFLUÊNCIA DO pH NA PRODUÇÃO DE CICLODEXTRINA GLICOSILTRANSFERASE (CGTase) POR Paenibacillus sp. F37
}

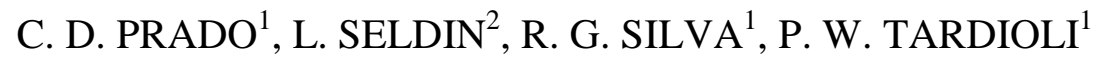 \\ ${ }^{1}$ Universidade Federal de São Carlos, Departamento de Engenharia Química \\ ${ }^{2}$ Universidade do Rio de Janeiro, Departamento de Microbiologia Geral, Instituto de \\ Microbiologia \\ E-mail para contato: professorcleitonquim@hotmail.com; pwtardioli@ufscar.br
}

\begin{abstract}
RESUMO - Ciclodextrina glicosiltransferase (CGTase - EC 2.4.1.19) é uma enzima de grande interesse industrial por catalisar a produção de ciclodextrinas (CDs) a partir de amido. CDs são oligossacarídeos cíclicos formados por unidades de glicose unidas por ligações $\alpha$-1,4-glicosídicas. As mais comuns possuem 6,7 ou 8 unidades de glicose, sendo denominadas de $\alpha-, \beta$ - e $\gamma-C D$, respectivamente. As CDs, por possuírem superfície externa hidrofílica e cavidade interna hidrofóbica, permitem o encapsulamento ao nível molecular de grande número de moléculas orgânicas, podendo melhorar a solubilidade e a estabilidade da molécula encapsulada. Devido à grande importância industrial da CGTase, esse trabalho teve por objetivo avaliar a produção dessa enzima por Paenibacillus sp. F37 em diferentes pH's de cultivo. A produção de CGTase foi realizada em meio Horikoshi com amido solúvel $1 \%, \mathrm{~m} / \mathrm{v}$ (indutor da enzima). Sob condições padronizadas $\left(37^{\circ} \mathrm{C}, 120 \mathrm{rpm}, 16 \mathrm{~h}\right.$ e meio de produção preparado em tampão tris- $\mathrm{HCl} 0,1 \mathrm{M}, \mathrm{pH}$ inicial 9,0) a atividade volumétrica obtida foi da ordem de $800 \mathrm{U} / \mathrm{L}$ (produtividade aproximada de $50 \mathrm{U} / \mathrm{L} \cdot \mathrm{h}^{-1}$ ). CGTase de Paenibacillus sp. $\mathrm{F} 37$ apresentou atividade máxima de ciclização em $50^{\circ} \mathrm{C}$ e pH 6,0. A enzima não purificada mostrou-se pouco estável nas condições ótimas de ciclização e nas condições de cultivo. A quantificação das CDs produzidas em reator batelada permitiu a classificação da enzima como uma $\beta$-CGTase.
\end{abstract}

\section{INTRODUÇÃO}

Ciclodextrina glicosiltransferase (1,4- $\alpha$-D-glicopiranosil transferase; EC 2.4.1.19; CGTase) é uma enzima, geralmente extracelular, da família das $\alpha$-amilases (Kuo et al., 2009), produzida por bactérias do gênero Bacillus (Nakamura e Horikoshi, 1976; Kitayska et al., 2011). Bactérias do gênero Paenibacillus têm sido reportadas como produtoras de CGTase (Charoensakdi et al., 2007; Kaulpiboon et al., 2010). Essas bactérias podem ser isoladas do solo, fontes vegetais, água, larvas de insetos, sedimentos marinhos contaminados com petróleo, etc. Produzem enzimas extracelulares e compostos antimicrobianos e antifúngicos inibidores de vários patógenos animais e vegetais (Lorentz, 2005). A CGTase catalisa a síntese de oligossacarídeos cíclicos, não redutores, conhecidos como ciclodextrinas (CDs), a partir de amido ou substratos similares (Szejtli, 1990; Fromming e Szejtli, 1994). As CDs mais comuns possuem 6, 7 ou 8 unidades de glicose, denominadas $\alpha-C D, \beta-C D$ ou $\gamma$-CD, respectivamente. As 
CGTases apresentam diferentes propriedades catalíticas, tais como, temperatura e $\mathrm{pH}$ ótimos, estabilidade térmica e o tipo de $\mathrm{CD}$ produzida.

As CDs possuem a capacidade de encapsulamento ao nível molecular de grande número de moléculas orgânicas (aromas, fragrâncias, fármacos, etc.), possuindo aplicação em indústrias de alimentos, cosméticos, fármacos e etc. (Tardioli et al., 2006). A inclusão molecular visa estabilizar compostos voláteis, desodorizar fármacos e alimentos, proteger princípios ativos contra oxidação e fotodegradação, aumentar a solubilidade de medicamentos insolúveis em água, mascarar gostos amargos de alimentos e fármacos, etc. (Kitayska et al., 2011). Muitos países têm aprovada a utilização de CDs ou derivados de CDs em alguns produtos, tais como, Voltaren oftálmico (complexo diclofenaco sódico/hidroxipropil $\gamma$-CD), Flogene (complexo Piroxicam $/ \beta-C D$ ), Nicorette (completo nicotina/ $\beta-C D$ ), gomas de mascar, xampus, etc. (Loftsson e Masson, 2001; Szejtli, 2004). Neste contexto, o objetivo desse trabalho foi avaliar a produção de CGTase por microrganismo do gênero Paenibacillus sp. por fermentação submersa em diferentes pH's e testar essa enzima na produção de CDs.

\section{MATERIAIS E MÉTODOS}

\subsection{Micro-organismo}

Paenibacillus sp. F37, gentilmente doada pela Profa. Dra. Lucy Seldin do Instituto de Microbiologia da UFRJ. Essa linhagem foi armazenada em criotubos a $80^{\circ} \mathrm{C}$ contendo $210 \mu \mathrm{L}$ de meio de cultura e $90 \mu \mathrm{L}$ de glicerol estéril/água $80 \%$ (v/v).

\section{Meio de cultivo}

O inóculo foi preparado em câmara incubadora a $37^{\circ} \mathrm{C}, 120 \mathrm{rpm}$ por $16 \mathrm{~h}$ a partir de um único criotubo em meio (volume total de $50 \mathrm{~mL}$ ) composto por peptona $\mathrm{G}\left(5 \mathrm{~g} . \mathrm{L}^{-}\right.$ 1, Acumedia ${ }^{\circledR}$ ), extrato de levedura (5 g.L-1, Himedia $\left.{ }^{\circledR}\right), \mathrm{K}_{2} \mathrm{HPO}_{4}$ (1 g.L ${ }^{-1}$ ), $\mathrm{MgSO}_{4} .7 \mathrm{H}_{2} \mathrm{O}$ (0,2 g.L'1), $\mathrm{Na}_{2} \mathrm{CO}_{3}(10$ g.L-1), pH 8,0. Uma alíquota (5ml) do inóculo foi transferido para $45 \mathrm{~mL}$ do meio de produção contendo amido solúvel (5 g.L'1, Qhemis $\left.^{\circledR}\right)$, peptona $\mathrm{G}\left(2,5\right.$ g.L-1), extrato de levedura $\left(2,5 \mathrm{~g} \mathrm{~L}^{-1} 1\right), \mathrm{K}_{2} \mathrm{HPO}_{4}\left(0,5\right.$ g.L $\left.{ }^{-1}\right)$, $\mathrm{MgSO}_{4} .7 \mathrm{H}_{2} \mathrm{O}$ (0,1 g.L-1) (Nakamura e Horikoshi, 1976). O pH foi variado dependendo do estudo. $\mathrm{O}$ meio foi mantido em câmara incubadora rotativa a $120 \mathrm{rpm}, 37^{\circ} \mathrm{C}$ por 48 h.

\subsection{Caracterização da enzima bruta}

CGTase bruta foi caracterizada quanto ao $\mathrm{pH}$ e temperatura ótima de ciclização e estabilidade térmica nas condições ótimas de ciclização e nas condições de cultivo do microrganismo. $\mathrm{O} \mathrm{pH}$ ótimo foi determinado por medidas de atividade de ciclização a $50^{\circ} \mathrm{C}, \mathrm{pH}$ 5,0 (citrato de sódio $0,1 \mathrm{M}$ ), 6,0 e 7,0 (fosfato de sódio 0,1M), 8,0 e 9,0 (Tris$\mathrm{HCl} 0,1 \mathrm{M})$ e 10,0 (Glicina/ $\mathrm{NaOH} 0,1 \mathrm{M})$, usando dextrina $1 \%(\mathrm{~m} / \mathrm{v})$ como substrato.

A temperatura ótima de ciclização foi determinada em $\mathrm{pH}$ 6,0 (citrato de sódio 0,1 M) na faixa de temperaturas de 30 a $70^{\circ} \mathrm{C}$, usando dextrina $1 \%(\mathrm{~m} / \mathrm{v})$ como substrato.

A estabilidade térmica foi avaliada nas condições de máxima atividade de ciclização $\left(50^{\circ} \mathrm{C}, \mathrm{pH} 6,0\right)$ e nas condições de cultivo do microrganismo $\left(37^{\circ} \mathrm{C}, \mathrm{pH} 9,0\right)$. 
As atividades residuais eram medidas de acordo com o protocolo padrão de atividade de ciclização, descrito na seção 2.4 .

\subsection{Atividade de ciclização}

A atividade de ciclização era calculada pela taxa de produção de $\beta-\mathrm{CD}$ a $50^{\circ} \mathrm{C}$, pH 8,0 (tampão tris- $\mathrm{HCl} 0,1 \mathrm{M}$, contendo $5 \mathrm{mM}$ de $\mathrm{CaCl}_{2}$ ), usando dextrina $1 \%(\mathrm{~m} / \mathrm{v})$ como substrato. A reação era iniciada pela adição de 0,5-1,0 $\mathrm{mL}$ de extrato enzimático em $20 \mathrm{~mL}$ de solução de dextrina. A reação era monitorada por $20 \mathrm{~min}$, coletando-se 2 $\mathrm{mL}$ do meio reacional a cada $5 \mathrm{~min}$ para dosagem colorimétrica de $\beta$-CD/fenolftaleína (Tardioli et al., 2006). Uma unidade de CGTase (U) foi definida como a quantidade de enzima que catalisa a formação de $1 \mu \mathrm{mol}$ de $\beta$-CD por min nas condições do ensaio.

\subsection{Produção de CDs}

CDs foram produzidas a $50^{\circ} \mathrm{C}, \mathrm{pH} 8,0$ (tampão tris- $\mathrm{HCl} 0,1 \mathrm{M}$ ), usando dextrina $5 \%(\mathrm{~m} / \mathrm{v})$, em reator do tipo batelada. A reação era iniciada pela adição de $10 \mathrm{~mL}$ de extrato enzimático bruto com aproximadamente $800 \mathrm{U} / \mathrm{L}$ a $100 \mathrm{~mL}$ de meio reacional (relação enzima/substrato $\approx 1,6 \mathrm{U} / \mathrm{g}$ ). A reação foi monitorada por $92 \mathrm{~h}$ por medidas de concentração CDs por métodos colorimétricos $\beta$-CD/fenolftaleína e $\gamma$-CD/verde de bromocresol (Tardioli et al., 2006).

As concentrações de CDs foram confirmadas por CLAE, usando um cromatógrafo Waters, equipado com detector de índice de refração (IR) ajustado em $45^{\circ} \mathrm{C}$ e auto-injetor ajustado em $4^{\circ} \mathrm{C}$. As amostras de CDs (padrões e meio reacional) eram filtradas em membrana de $0,2 \mu \mathrm{m}$ e um volume de $20 \mu \mathrm{L}$ era injetado em uma coluna Shodex Sugar KS-802 a $80^{\circ} \mathrm{C}$. As CDs $(\alpha-, \beta-$, e $\gamma$-CD) eram separadas com água como fase móvel a uma vazão de $1 \mathrm{~mL} / \mathrm{min}$. Os tempos de retenção de cada $\mathrm{CD}$, bem como suas curvas padrões foram determinados com padrões de $\alpha-, \beta-$, e $\gamma$-CD (Sigma-Aldrich), maltose e glicose (Synth). A análise de CDs a partir do meio reacional de produção sofria interferência por dextrinas residuais. Assim, as amostras $(1 \mathrm{~mL})$ eram tratadas com $0,5 \mathrm{~mL}$ de amiloglicosidase de Aspergillus oryzae (AMG $300 \mathrm{~L}^{\mathrm{TM}}$, Sigma-Aldrich) a $60^{\circ} \mathrm{C}$, por $1 \mathrm{~h}$, a fim de se hidrolisar as dextrinas residuais a glicose e maltose, principalmente.

\section{RESULTADOS E DISCUSSÃO}

\subsection{Produção de CGTase}

De acordo com a literatura (Prado et al. 2002; Matioli et al. 2000) embora as máximas produções de CGTase são obtidas em pH's alcalinos, optou-se por realizar cultivos de Paenibacillus sp. F37 em diferentes pH's iniciais $(6,0$ a 11,0) sem o tamponamento do meio. Os resultados de atividade enzimática após 24 h de cultivo são mostrados na Figura 1(a).

Os resultados mostraram que as maiores atividades volumétricas ocorreram em valores alcalinos de $\mathrm{pH}(9,0$ a 11,0), corroborando com trabalhos previamente reportados (Mahat et al., 2004; Matioli et al. 2000). Entretanto, observou-se em todos os cultivos uma grande diminuição de $\mathrm{pH}$ após $24 \mathrm{~h}$, atingindo valores finais de 5,5 a 6,0 . 


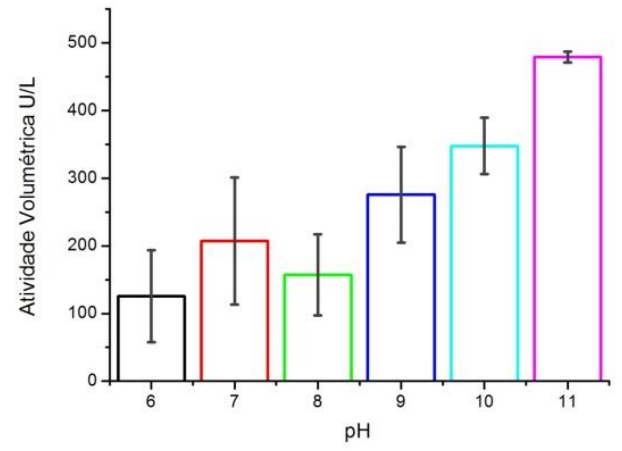

(a)

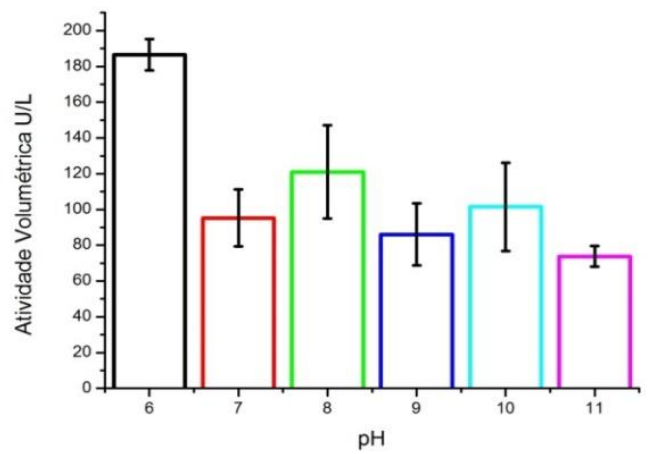

(b)

Figura 1 - Atividade enzimática de CGTase em cultivos de Paenibacillus sp. F37 a $37^{\circ} \mathrm{C}$.

(a) em diferentes valores inicias de $\mathrm{pH}$ e (b) em $\mathrm{pH}$ do meio tamponado em diferentes valores.

Diante dessa diminuição de $\mathrm{pH}$, realizaram-se cultivos com meio de produção preparados em pH's 8,0, 9,0 (Tris- $\mathrm{HCl} 0,1 \mathrm{M})$ e $10,0\left(\mathrm{Na}_{2} \mathrm{CO}_{3} 0,1 \mathrm{M}\right)$. Nestes cultivos (resultados não mostrados), o maior valor de atividade enzimática (aproximadamente $700 \mathrm{U} / \mathrm{L}$ após $16 \mathrm{~h}$ ) foi obtido em $\mathrm{pH}$ inicial 9,0. Entretanto, embora o pH tenha permanecido por mais tempo em valores alcalinos, após $16 \mathrm{~h}$ reduziu-se para 6,5.

Novos cultivos foram realizados com meio de produção preparados em tampões com maior força iônica para garantir o tamponamento do meio no $\mathrm{pH}$ desejado. Os cultivos foram realizados em $\mathrm{pH}$ 's 6,0, 7,0 e 8,0 (fosfato de sódio 0,5M), 9,0 (Tris- $\mathrm{HCl}$ $0,5 \mathrm{M}), 10,0\left(\mathrm{Na}_{2} \mathrm{CO}_{3} 0,1 \mathrm{M}\right)$ e 11,0 (glicina/NaOH $\left.0,5 \mathrm{M}\right)$. Nessas condições, os meios de cultivo se mantiveram tamponados nos $\mathrm{pH}$ 's desejados.

A Figura 1(b) mostra que para os cultivos em meios tamponados, a máxima produção de CGTase foi obtida em pH 6,0. Entretanto, as atividades volumétricas foram inferiores às obtidas em meios não tamponados (Figura 1a).

O estudo da influência do $\mathrm{pH}$ no meio de produção de CGTase por Paenibacillus sp. F37 mostrou que a maior produção da enzima ocorreu $\mathrm{pH}$ inicial 9,0, com esse decrescendo para valores neutros ao longo do cultivo ( $\mathrm{pH}$ final 6,5). Esse comportamento levou-se a suspeitar que o crescimento do microrganismo e a produção da enzima eram favorecidos em $\mathrm{pH}$ alcalino, enquanto a estabilidade da enzima ao $\mathrm{pH}$ era favorecida em $\mathrm{pH}$ neutro. Dessa forma, realizaram-se cultivos em $\mathrm{pH} \mathrm{9,0} \mathrm{com} \mathrm{os}$ meios de produção preparados em tampão tris- $\mathrm{HCl}$ com duas concentrações distintas: 0,1 M para manter o $\mathrm{pH}$ em valores alcalinos nas primeiras horas de cultivo e em valores neutros no restante, e $0,5 \mathrm{M}$ para garantir o tamponamento durante todo $\mathrm{o}$ cultivo. O resultados mostraram (Figura 2) que após $16 \mathrm{~h}$ de cultivo a primeira condição conduziu a uma atividade volumétrica em torno de $800 \mathrm{U} / \mathrm{L}$, aproximadamente 4 vezes superior à obtida na segunda condição. 


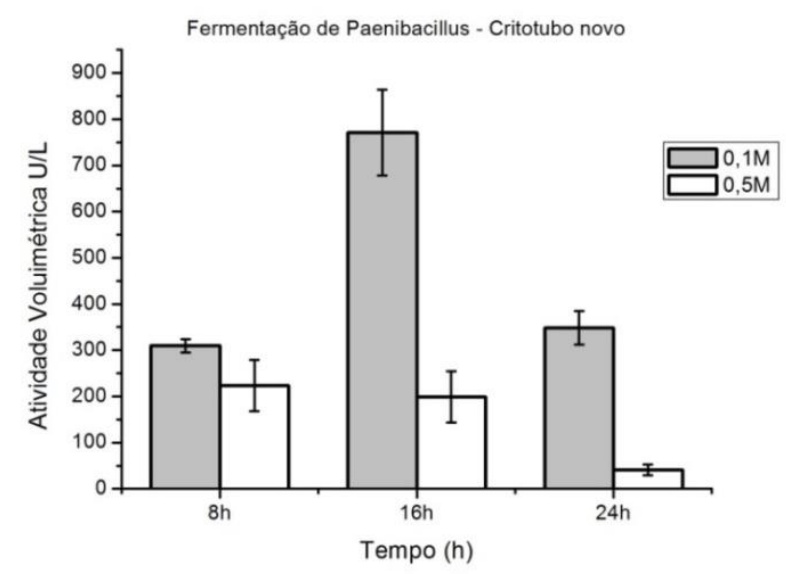

Figura 2 - Atividade enzimática de CGTase em cultivos de Paenibacillus sp. F37 a $37^{\circ} \mathrm{C} \mathrm{em}$ pH 9,0 em tampão Tris-HCl 0,1M e Tris-HCl 0,5M.

Conclui-se que a melhor condição estabelecida nesse trabalho para produção de CGTase de Paenibacillus sp. F37 foi iniciar o cultivo em pH 9,0, permitindo que esse decresça a valores neutros ao longo do cultivo. Essa condição pode ser alcançada preparando-se o meio de cultivo em tampão tris- $\mathrm{HCl} 0,1 \mathrm{M}$, mantendo-se o meio por 16 h a $37^{\circ} \mathrm{C}, 120 \mathrm{rpm}$.

\subsection{Caracterização da CGTase}

As atividades de ciclização a $50^{\circ} \mathrm{C}$ e diferentes valores de $\mathrm{pH}$ mostraram (Figura 3a) máxima atividade em $\mathrm{pH}$ 6,0, similarmente ao $\mathrm{pH}$ ótimo de algumas CGTases produzidas por outros microrganismos: CGTase de Bacillus sp. alcalophilus E16 (Alves-Prado, 2000), CGTase de Bacillus sp. G1 (Sian et al., 2005) e CGTase de Paenibacillus macerans (Li et al., 2010).

As atividades de ciclização em $\mathrm{pH}$ 6,0 e diferentes temperaturas mostraram (Figura 3b) máxima atividade em $50^{\circ} \mathrm{C}(1200 \mathrm{U} / \mathrm{L})$. Cucolo et al., 2006, reportaram temperatura ótima de $55^{\circ} \mathrm{C}$ para CGTase de Bacillus sp. alcalofílico E16. Li et al., 2010 obtiveram temperatura ótima de $45^{\circ} \mathrm{C}$ para CGTase de Paenibacillus macerans.

As Figuras 4(a) e 4(b) mostram os resultados de estabilidade térmica da CGTase de Paenibacillus sp. F37 a 50 $\mathrm{C}, \mathrm{pH}$ 6,0 (condições de máxima atividade de ciclização) e $37^{\circ} \mathrm{C}$, pH 6,0 (condições de cultivo), respectivamente. Observa-se baixa estabilidade da enzima em ambas as condições (meias-vidas de 2 e $3 \mathrm{~h}$, respectivamente). É importante ressaltar que a baixa estabilidade da enzima nas condições de cultivo (perda de quase $80 \%$ de atividade em $5 \mathrm{~h}$ ) corrobora com a hipótese de que é importante iniciar o cultivo do microrganismo em $\mathrm{pH}$ alcalino para favorecer o seu crescimento e, por conseguinte, a produção da enzima, mas manter o $\mathrm{pH}$ ao longo do cultivo em valores próximos da neutralidade para manter a atividade da enzima secretada. 


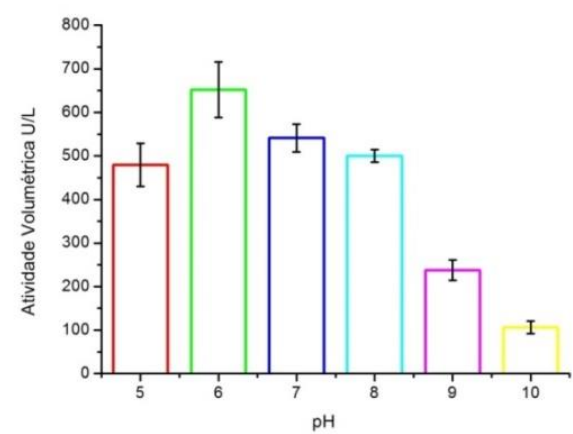

(a)

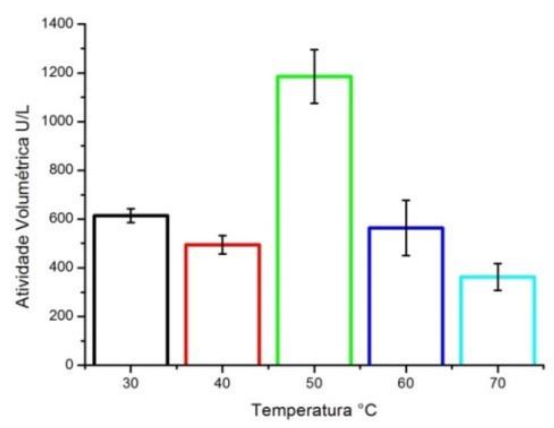

(b)

Figura 3 - Atividade volumétrica de CGTase produzida por Paenibacillus sp. F37. (a) atividade volumétrica a $50^{\circ} \mathrm{C}$ e diferentes valores de $\mathrm{pH}(5,0$ a 10,0). (b) atividade volumétrica a $\mathrm{pH} 6,0$ e diferentes valores de temperatura $\left(30\right.$ a $\left.70^{\circ} \mathrm{C}\right)$.

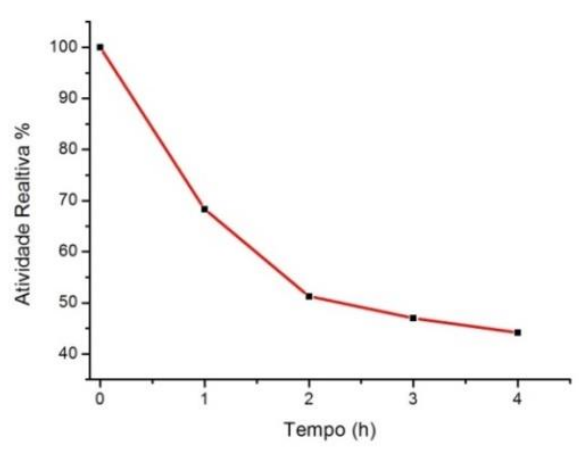

(a)

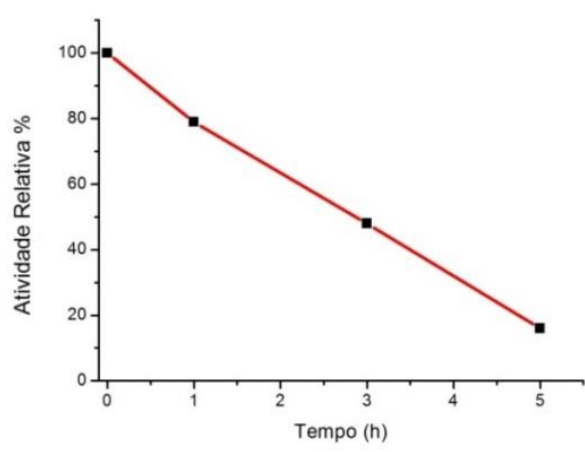

(b)

Figura 4 - Estabilidade de CGTase de Paenibacillus sp. F37 (atividade inicial de ciclização foi considerada $100 \%$ ). (a) $\mathrm{pH} 6,0$ e $50^{\circ} \mathrm{C}$ e (b) $\mathrm{pH} 9,0$ e $37^{\circ} \mathrm{C}$

\subsection{Produção de CDs}

Trabalhos reportados na literatura (Larsen et al, 1998) indicam que CGTase produzida por bactérias do gênero Paenibacillus é preferencialmente produtora de $\beta$ $\mathrm{CD}$, sendo assim classificada como $\beta$-CGTase. A fim de classificar a CGTase produzida por Paenibacillus sp. F37 foram realizados ensaios de produção de CDs em reator batelada, a $50^{\circ} \mathrm{C}$ e $\mathrm{pH} 8,0$, usando solução de dextrina $5 \%(\mathrm{~m} / \mathrm{v})$ como substrato. As concentrações de $\beta$ - e $\gamma$-CD ao longo da reação foram monitoradas por dosagem colorimétrica, e as concentrações finais, após $92 \mathrm{~h}$ de reação, foram confirmadas por cromatografia líquida. 


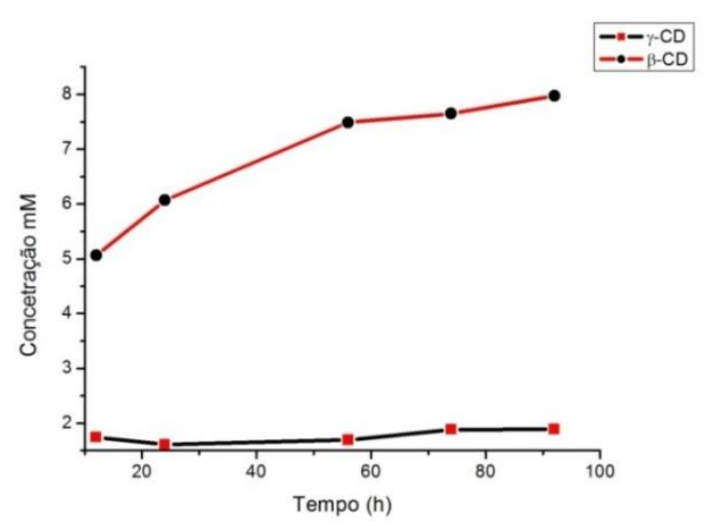

Figura 5 - Produção de $\mathrm{CDs}$ a $50^{\circ} \mathrm{C}$, usando como substrato solução de dextrina $5 \%, \mathrm{~m} / \mathrm{v}$, preparada em tampão Tris- $\mathrm{HCl} 0,1 \mathrm{M}, \mathrm{pH} 8,0,92$ h de reação, relação enzima/substrato de $1,6 \mathrm{U} / \mathrm{g}$.

A Figura 5 mostra que a enzima produziu preferencialmente $\beta-C D$, sendo a concentração desta $\mathrm{CD}(7,9 \mathrm{mM})$ aproximadamente 4 vezes maior do que a de $\gamma-\mathrm{CD}$ $(1,8 \mathrm{mM})$ após $92 \mathrm{~h}$ de reação.

A metodologia adotada para a quantificação de CDs por CLAE permitiu somente a identificação de $\beta-\mathrm{CD}$, sendo a concentração desta igual a $8,6 \mathrm{mM}$, valor este em torno de $10 \%$ superior ao encontrado por dosagem colorimétrica. Não foi possível quantificar as outras CDs $(\alpha-$ e $\gamma-C D)$ por serem produzidas em baixa quantidade e o método cromatográfico não ter permitido a separação adequada dessas CDs. Esta técnica requer estudos adicionais para uma separação adequada das CDs produzidas.

Com base nos resultados de dosagem colorimétrica pode-se classificar a CGTase produzida por Paenibacillus sp. F37 como $\beta-$ CGTase. CGTases produtoras de $\beta-C D$ são atrativas do ponto de vista industrial, pois devido a baixa solubilidade desta $C D$, esta é facilmente separada e purificada por cristalização a partir do meio de produção (Pongawasdi e Yagisawa,1987, Tardioli, 1998).

\section{CONCLUSÃO}

O cultivo de Paenibacillus sp. F37 em câmara incubadora rotativa (120 rpm) a $37^{\circ} \mathrm{C}$ atingiu maiores atividades volumétricas de CGTase em meio de produção contendo amido solúvel $1 \%(\mathrm{~m} / \mathrm{v})$, preparado em tampão tris- $\mathrm{HCl} 0,1 \mathrm{M}$, com pH inicial de 9,0, decrescendo para 6,2 após 16 h de cultivo. A enzima não purificada apresentou máxima atividade de ciclização (formação de $\beta-\mathrm{CD}$ ) a $50^{\circ} \mathrm{C}, \mathrm{pH} 6,0$, tendo nessas condições baixa estabilidade (meia-vida em torno de $2 \mathrm{~h}$ ). Igualmente, baixa estabilidade foi verificada a $37^{\circ} \mathrm{C}$ e $\mathrm{pH} 9,0$ (perde de $80 \%$ da atividade em $5 \mathrm{~h}$ ), mostrando que um cultivo de Paenibacillus sp. F37 com pH controlado em 9,0 conduziria a grande perda de atividade por inativação da enzima. A produção de CDs em reator batelada permitiu a classificação da enzima como uma $\beta$-CGTase, pois o meio de produção apresentou 74\% (base mássica) de $\beta-\mathrm{CD}$. 


\section{REFERÊNCIAS}

ALVES-PRADO, H.F. Estudo da produção de ciclodextrina glicosiltransferase (CGTase) por Bacillus sp alcalofílico. Dissertação de Mestrado, Universidade Estadual Paulista (UNESP), Rio Claro, 144f, 2000.

CHAROENSAKDI, R. et al. Cloning and expression of cyclodextrin glycosyltransferase gene from Paenibacillus sp.T16 isolated from hot spring soil in northern Thailand. Journal of Biochemistry and Molecular Biology, 40(3), 333-340, 2007.

CUCOLO, G.R.; ALVES-PRADO, H.F.; GOMES, E.; SILVA, R. Otimização da produção de CGTase de Bacillus alcalophilus E16 em polvilho doce em fermentação submersa, São José do Rio Preto, v.9, n.3, p. 201-208, jul./set 2006.

FRÖMMING, K.H.; SZEJTLI, J. Cyclodextrins in Pharmacy. Dordrecht, Kluwer Academic Publishers, pp. 1-81, 1994.

KAULPIBOON, J. et al. Expression and characterization of a fusion protein-containing cyclodestrin glycosyltransferase from Paenibacillus sp. A11. Journal of Basic Microbiology, 50(5), 427-435, 2010. KITAYSKA, T. et al. Purification and properties of a new Thermostable cyclodextrin glucanotransferase fromBacillus pseudalcaliphilus 8SB. Applied Biochemistry and Biotechnology, 165, 1285-1295, 2011.

KUO, C.C. et al. Production of cyclodextringlucanotransferease from an alkalophilic Bacillus sp. by pH-stat fed-batch fermentation. Biotechnology Letters, 31, 1723-1727, 2009.

LARSEN, K.L. et al. Purification and characterisation of cyclodextrin glycosyltransferase from Paenabacillus sp. F8. Carbohydrate Research, 310, 211-219, 1998.

LI, Z. et al. Extracellular expression and biochemical characterization of alpha-cyclodextrin glycosyltransferase from Paenibacillus macerans. In Carbohydrate Reserch, v. 345, Elsevier Science Publishers Ltd, pp. 886-892, 2010.

LOFTSSON, T.; MASSON, M. Cyclodextrins in topical drug formulations: theory and practice. Int. J. Pharm., 225, 15-30, 2001.

LORENTZ, R.H. Seleção de isolados de Paenabacillus sp. com atividade enzimática e antimicrobiana. Universidade Federal do Rio Grande do Sul, Faculdade de Agronomia. Dissertação, Porto Alegre, 2005.

MAHAT, M. K. et al. Production of cyclodextrin glucanotransferase (CGTase) from alkalophilic Bacillus sp. TS1-1: media optimization using experimental design. Appl. Enzyme and Microbial Technology., 467-473, 2004.

MATIOLI, G. et al. Estudos de parâmetros que influenciam na produção da enzima CGTase de Bacillus firmus, cepa no 37. In: Acta Scientarium, v. 22, pp 311-316, 2000.

NAKAMURA, N; HORIKOSHI, K. Characterization and some cultural conditions of a cyclodextrin glycosyltransferase-producing alkalophilic Bacillus sp. Agric. Biol. Chem., 40, 753-757, 1976.

PONGAWASDI, P.; YAGISAWA, M. Screnning and Indentification of a Cyclomaltodextrin Glucanotransferase-Producing Bacteria. Journal Fermentation Technology, v. 65, n. 4, pp. 463-467. 1987.

PRADO, H.F.A; HILARIO E; SILVA, E.G. Seleção de Microrganismos Produtores de ciclodextrina glicosiltransferase (CGTase) e produção e caracterização da enzima. Braz. J. Technol, n 98, 2002.

SIAN, H.K. et al. Purification and characterization of cyclodextrin glucanotransferase from alkalophilic Bacillus sp G1. Process Biochemistry.v. 40, n. 1-2, p. 1101-1111, 2005.

SZEJTLI, J. The cyclodextrins and their applications in biotechnology. In: Carbohydrate Polymers, v. 12, Elsevier Science Publishers Ltd, pp. 375-392, 1990.

TARDIOLI, P.W.; ZANIN, G.M.; MORAES, F.F. Characterization of Thermo anaerobactercy clomaltodextrin glucanotransferase immobilized on glyoxyl-agarose, Enzyme Microb. Technology.v.39, p.1270-1278, 2006.

TARDIOLI, Paulo. Produção de ciclodextrinas em reator de leito fluidizado com a enzima ciclodextrina glicosiltransferase Imobilizada. 1998. 194 f. Dissertação de Mestrado em Engenharia Química, Departamento de Engenharia Química, Universidade Estadual de Maringá, Maringá, 1998.

SZEJTLI, J. Past, present, and future of cyclodextrin research. Pure Appl. Chem., 76, 1825-1845, 2004. 